\title{
Ein Traumberuf?
}

\section{Michel Matter}

Dr. med., Departementsverantwortlicher Dienstleistungen und Berufsentwicklung

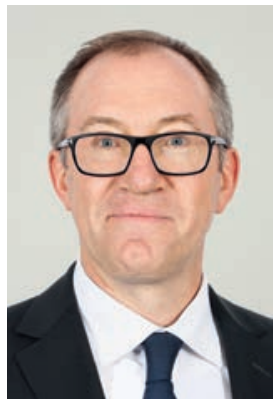

Häufig kommen Eltern auf mich zu und fragen, ob die Entscheidung ihres Kindes, Medizin zu studieren, richtig ist. Jahr für Jahr ist unser Beruf immer stärkerem Druck ausgesetzt und macht Schlagzeilen - mitunter zu Recht, häufig aber auch zu unrecht. Trotzdem ist meine Antwort ein klares "Ja»! Was zählt, ist das, was man im Herzen trägt. Die Lust muss im Vordergrund stehen, der Wille, seinem Nächsten zu helfen und Gutes zu tun. Unser Beruf ist in erster Linie ein spannendes menschliches Abenteuer. Er bleibt jedoch eng verbunden mit einem sich schnell entwickelnden Technologiefeld und der ständigen Betrachtung des Menschen und seines Platzes im Verlauf des Lebens und im Angesicht des Todes. Mit anderen in Kontakt zu stehen, ihnen unser Wissen zu vermitteln, ihnen bei ihrer Therapie zu helfen, ihnen zuzuhören und selbst ihre intimsten Geheimnisse zu teilen ist bereichernd und beflügelnd. Dennoch ist unser Arbeitsalltag nicht nur eitel Sonnenschein. Das Hinterfragen unseres Berufs und seiner Zukunft ist wichtig, das greifbare Leiden der Dienstleistungserbringer im Pflegebereich und die Ernüchterung über den Berufsalltag werden zunehmend öffentlich zur Sprache gebracht.

Aufgabe des Departements Dienstleistungen und Berufsentwicklung ist es, die aufgeworfenen Fragen zu analysieren und $\mathrm{zu}$ berücksichtigen. Aus diesem Grund ist unsere Umfrage bei Studierenden, durch die wir ihre Hoffnungen und Erwartungen in Erfahrung bringen und verstehen möchten, von wesentlicher Bedeutung. Einerseits soll die FMH, ihre Rolle und ihre Tätigkeiten, vorgestellt werden, vor allem aber sollen andererseits auch die Vorstellungen und Wünsche der Generation der Millennials berücksichtigt werden. Gleichzeitig werden die Arbeitsbedingungen unserer jungen Kolleginnen und Kollegen während ihrer Weiterbildung unmittelbar vom VSAO überprüft. Die ärztlichen, chirurgischen und kantonalen Vereinigungen und Organisationen erhalten häufig Rückmeldungen von Ärztinnen und Ärzten betreffend die Schwierigkei- ten, mit denen sie im Berufsalltag konfrontiert sind. Durch diese Daten, Erwägungen und Antworten muss sich ein lückenloses Bild ergeben, um einen genauen Überblick über die Ausübung unseres Berufs in der Schweiz zu erhalten. Wir müssen für alle Leistungserbringer bestmögliche Arbeitsbedingungen sicherstellen, und zwar natürlich nicht nur für die Ärztinnen und Ärzte, sondern für alle Berufsgruppen.

Die Politik will die Anzahl an Medizinern steuern, indem sie niedergelassene Ärztinnen und Ärzte auf eine fixe Zahl begrenzt. Wie sollten wir Studierenden und jungen Kolleginnen und Kollegen erklären, dass sie

\section{Beim Entscheid für den Arztberuf muss der} Wille, seinem Nächsten zu helfen und Gutes zu tun, im Vordergrund stehen.

nach 10 bis 12 Jahren Studium und Ausbildung auf Staatskosten nicht mehr die Möglichkeit haben sollen, sich zu entscheiden, ob sie in eine Praxis gehen, oder in den Spitälern bleiben wollen? Dies ergibt ebenso wenig Sinn wie die vom Bundesrat erklärte Absicht, ein Globalbudget einzuführen, das zu Rationierungen im Gesundheitswesen führt, wie es in Deutschland dramatisch der Fall ist. Sollten solche Entscheide wirklich gefällt werden, muss man sie anfechten.

Also ja, Medizin zu studieren ist ein Traum, und wir müssen unseren Beitrag dazu leisten. Es ist unsere Aufgabe, sicherzustellen, dass die Arbeitsbedingungen und das Gleichgewicht zwischen Zeit für die Patienten, Verwaltung und Privatleben während der gesamten beruflichen Laufbahn von Ärztinnen und Ärzten optimiert werden können. Das Wachstum des Frauenanteils in der Medizin sowie auch der Eintritt der Generation der Millennials ins Berufsleben beschleunigt nicht nur das Neuerwägen der Situation, sondern es stellt auch eine grosse Chance dar, nicht nur für unseren Beruf und im Hinblick darauf, das Leben von Medizinern neu zu gestalten, sondern für unser gesamtes Land. 